\title{
Development and Validation of Extraction Method for the Determination of UC781 in Cervicovaginal Fluid
}

\author{
Naser L. Rezk ${ }^{1,2,3^{*}}$, Zhiqing Qiao ${ }^{1}$, Majed Jeriasy ${ }^{3}$ \\ ${ }^{1}$ Eshelman School of Pharmacy, Division of Pharmacotherapy and Experimental Therapeutics, \\ University of North Carolina, Chapel Hill, USA \\ ${ }^{2}$ Andor Labs, Durham, North Carolina, USA \\ ${ }^{3}$ King Abuallah International Medical Research Center, Riyadh, KSA \\ Email: "naser21@gmail.com, ${ }^{*}$ rezkab@ngha.med.sa, ${ }^{*}$ rezkn@andorlabs.com
}

Received October 25, 2013; revised November 26, 2013; accepted December 1, 2013

Copyright (C) 2013 Naser L. Rezk et al. This is an open access article distributed under the Creative Commons Attribution License, which permits unrestricted use, distribution, and reproduction in any medium, provided the original work is properly cited.

\begin{abstract}
Liquid chromatography plays the important and critical role in the field of clinical pharmacology of evaluating drugs in biological matrices. Studying antiretroviral drugs in the female genital tract has important implications for using drugs as a vaginal microbicide for prevention of HIV-1 sexual transmission. Accurate measurement of drug levels is extremely important in optimizing drug concentration in gel formulation. Extracting drugs from small volumes of viscous, proteinaceous substances like cervicovaginal fluid (CVF) is a practically challenging process. The proposed method was designed to introduce procedure for sample collection and drug extraction procedure for CVF matrix before the chromatographic separation. Based on this extraction method, we validated a reverse phase high performance liquid chromatography with electrospray ionization mass spectrometry assay in order to quantify UC781 in female genital tract compartment. The LC-MS method was validated based on a novel extraction technique which proved to be efficient in reducing analyte degradation with an average extraction efficiency of $72 \%$. This method is accurate, demonstrating an average accuracy over three QC $(\mathrm{n}=30)$ concentrations ranging from $99.9 \%$ to $106.1 \%$. Average precision within-day and between-day ranged from $3.1 \%$ to $10.2 \%$ and $5.1 \%$ to $6.4 \%$, respectively. We demonstrated that the analyte was able to maintain its stability under various conditions using this extraction method. The sample preparation, extraction, and the powerful liquid chromatography and mass spectrometry can readily be applied for accurate quantification of similar drugs in CVF.
\end{abstract}

Keywords: HIV-Prevention; Cervicovaginal Fluid; Extraction; LC-MS; Nonnucleoside Reverse Transcriptase Inhibitor

\section{Introduction}

Sexual transmission of HIV is the principal mode of spread of HIV throughout the world [1]. The majority of HIV-1 infections are acquired sexually, and interventions to prevent sexual transmission are urgently needed to curb the growth of the HIV pandemic [2].

Methods to reduce or prevent sexual transmission of HIV-1 are urgently needed to sharply reduce the global HIV-1 epidemic [3]. Understanding the pharmacokinetics of drugs in human body compartments, such as the female genital tract, is especially important [4]. Among several classes of HIV inhibitors, many drugs belong to non-nucleoside reverse transcriptase inhibitors (NNRTIs). The thiocarboxanilide ((N-[4-chloro-3-(3-methyl-2-bu-

${ }^{*}$ Corresponding author. tenyloxy) phenyl]-2-methyl-3-furancarbotthioamide) or UC-781 (Figure 1(a)) is ranked among the most potent NNRTI's [5-8]. Currently, UC781 is being investigated as a prevention therapy $[9,10]$. The highly potent nonnucleoside reverse transcriptase inhibitor UC781 has been tested as a safe vaginal microbicide gel formulation for preventing the sexual transmission of HIV-1 virus [10]. To investigate whether UC781 retained anti-infective activity following exposure to the female genital tract, we developed the assay for the analysis UC781 levels and antiviral activity in cervicovaginal fluid (CVF). Based on our LC-ESI-MS method [11] for sensitive and accurate determination of UC781 in blood plasma, we modified the extraction method to fit with the (CVF) matrix.

An optimal extraction method for female genital tract secretion must include the release of drug candidate from 
UC781<smiles>CC(C)=CCOc1cc(NC(=S)c2ccoc2C)ccc1Cl</smiles>

(a)

F2951<smiles></smiles>

(b)

Figure 1 (a) Chemical structure of UC781; (b) Chemical structure of F2591 (IS).

the viscous proteinaceous substances [12] like CVF. In this manuscript, the primary objective of this work is to develop and optimize an extraction method for a sample clean-up procedure to fit with CVF. The second objective is to validate the procedure to be a standard assay for quantification of drug concentration in CVF.

\section{Materials and Methods}

\subsection{Chemicals}

UC781 (purity 98.7\%) was supplied by Regis Technologies, Inc (Morton Grove, IL, USA). F2951 (purity 99.2\%), used as internal standard, was supplied by Chemtura, Technology Center (Guelph, Ontario, Canada). Tetrahydrofuran (purity 99.9\%) was purchased from Aldrich (St. Louis, MO, USA). HPLC-grade reagents and chemicals were purchased from Fisher Scientific (Norcross, GA, USA). Purified compressed nitrogen gas was obtained from National Welders Supply (Charlotte, NC, USA).

\subsection{Equipment}

An Eppendorf® Positive Displacement contaminationfree pipette (PDP), adjustable ranges $1 \mu \mathrm{l}-20 \mu \mathrm{l}$ and Positive Displacement Tips obtained from (Eppendorf North America, Washington DC, USA). This pipette functions according to the positive-displacement principle in conjunction with the special Positive Displacement Tip (Figure 2). An Eppendorf 5415D centrifuge (Eppendorf AG, Hamburg, Germany) was used during sample preparation. The high-performance liquid chromatography (HPLC) system consisted of an Agilent Technologies (Wilmington, DE, USA) HP1100 binary pump, degasser, and thermostatic auto sampler (programmed at $4^{\circ} \mathrm{C}$ ). The HPLC system was connected to a 1100 Series Mass Spectrometer. Positive electrospray ionization was the mode used for analytical compounds. Data analysis was performed using HP ChemStation software (Version A.09.03) run on a Dell computer (Windows 2000 Professional operating system).

\subsection{Blank Vaginal Fluid Collection and Preparation}

The biological matrix, vaginal secretion fluid (CVF) was collected from five healthy subjects. The collection of CVF has been approved by the institutional review board (IRB) at the University of North Carolina at Chapel Hill (UNC) according the Federal-Wide Assurance (FWA) \#4801, the IRB approval \# (45 CFR 46 CFR 46.110), and all volunteers/patients gave informed consent prior to study participation.

Before spiking drug to the pooled CVF, the vaginal secretion was diluted 1:3 in normal saline. Because the volume of CVF varies from subject to subject, a similar approach was used on the patient sample directly after the collection of CVF secretion and before storage. Based on the amount of collected CVF, the PDP adjustable positive displacement pipette should be adjusted on 5 or 10 or 15 or 20 , then pipette the possible volume, wipe the narrow tip. Then transfer the aspirate into a clean tube containing three times the volume of the aspirate.

\subsection{Preparation of Standards}

A total of $5.066 \mathrm{mg}$ of UC781 (molecular weight 335.82)

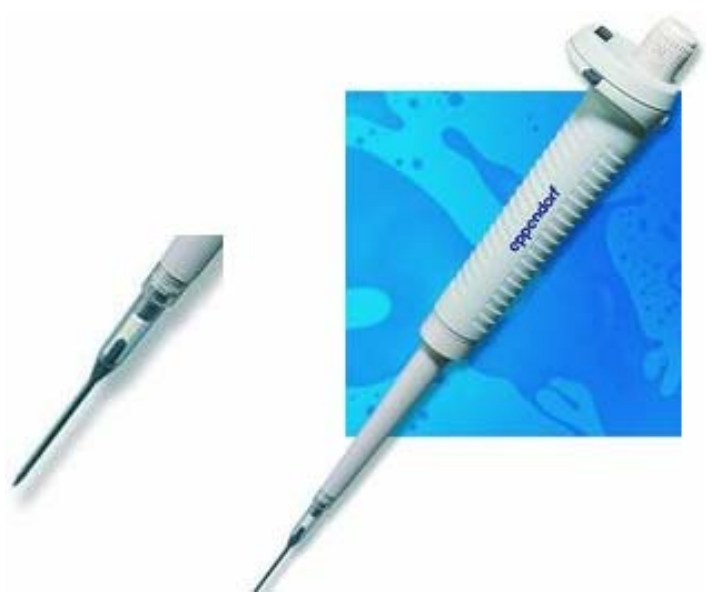

Figure 2. Positive displacement with a special tip. 
powder with a purity of $98.7 \%$ was accurately weighed and dissolved in $5 \mathrm{~mL}$ of methanol to produce a final concentration of $1 \mathrm{mg} \cdot \mathrm{mL}^{-1}$. The master stock solution was prepared by diluting a stock of $1 \mathrm{mg} \cdot \mathrm{mL}^{-1}$ to 500 $\mu \mathrm{g} \cdot \mathrm{mL}^{-1}$ in $50 \%$ HPLC-grade methanol/HPLC-grade water. This $500 \mu \mathrm{g} \cdot \mathrm{mL}^{-1}$ master solution was used to prepare six working solutions in methanol/HPLC-grade water $(1: 1)$ at concentrations of $0.5-500 \mathrm{mg} \cdot \mathrm{mL}^{-1}$ of UC781. CVF calibration samples (50, 100, 500, 1000, $5000,10,000,25,000$ and $50,000 \mathrm{ng} \cdot \mathrm{mL}^{-1}$ of UC781) were prepared by using a 1:10 dilution of the respective working solutions to blank CVF. From an additional 500 $\mathrm{ng} \cdot \mathrm{mL}^{-1}$ working stock solution, concentrations of 1.5 $\mu \mathrm{g} \cdot \mathrm{mL}^{-1}, 15 \mu \mathrm{g} \cdot \mathrm{mL}^{-1}$ and $150 \mu \mathrm{g} \cdot \mathrm{mL}^{-1}$ of UC781 were prepared in methanol/HPLC-grade water $(1: 1)$. CVF quality control samples at concentrations of 150, 1500, and $15,000 \mathrm{ng} \cdot \mathrm{mL}^{-1}$ of UC781 were prepared using a 1:10 dilution of their respective working solutions to blank CVF.

\subsection{Internal Standard (IS) Preparation}

F2951 (Figure 1(b)) powder (5.187 mg; purity 99.2\%) was dissolved in $3 \mathrm{~mL}$ methanol, then complete the volume of methanol $(5.0 \mathrm{~mL})$ to achieve a final concentration of $1.0 \mathrm{mg} \cdot \mathrm{mL}^{-1}$ (stock solution). From this solution, an aliquot was diluted in HPLC-grade acetonitrile to a final concentration of $20.0 \mathrm{ng} / \mathrm{mL}$ (working solution). Small aliquots of working solution must be stored at $-20^{\circ} \mathrm{C}$.

\subsection{Extraction Procedure}

The extraction of UC781 from the CVF matrix was performed with liquid-liquid extraction, using diethylether. Prior to extraction, CVF protein was precipitated. The precipitation occurred by adding $10 \mu \mathrm{L}$ aliquot of CVF blank, calibrators, and QCs into $2.0 \mathrm{~mL}$ tube containing $20 \mu \mathrm{L}$ the internal standard made in acetonitrile. All matrix aliquots were pipette using positive displacement pipette with narrow end tip (Figure 2) which easy to wipe off the remaining outside sticky fluid for better accuracy. The tips are narrow, transparent and long enough to monitor the volume. The solutions were mixed via vortex-mixing for 30 seconds. After vortex-mixing, $10 \mu \mathrm{l}$ of $(0.1 \mathrm{~N}) \mathrm{NaOH}$ was added to each tube, followed by $1.7 \mathrm{~mL}$ of the extraction liquid. All tubes were immediately capped and gently mixed for $30 \mathrm{~min}$ at low speed. All tubes were placed in a dry ice/acetone bath for approximately 1 minute; the aqueous portion of the sample was frozen and the organic layer was immediately transferred to a centrifuge tube and evaporated until dry under a nitrogen stream at $35^{\circ} \mathrm{C}$ for approximately 8 mints. Finally, the residue was reconstituted with $100 \mu \mathrm{L}$ of methanol/water $(50 / 50)$. The resulting solutions were carefully vortexed for $30 \mathrm{~s}$ and centrifuged at 14,000 rcf for 3 mints. The supernatants were transferred to $200 \mu \mathrm{L}$ HPLC micro-vials (Agilent Technologies), and $10 \mu \mathrm{L}$ of each sample was injected for LC-MS analysis.

\subsection{Chromatography Separation Conditions}

1) High performance liquid chromatography conditions

Chromatographic separation was performed using gradient elution. Separation was conducted using an Allure C-18 $(100 \times 2.1 \mathrm{~mm}, 3.0 \mu \mathrm{m}$ particle size, Restek, Bellefonte, PA, USA) analytical column with an Allure C-18 $(10 \times 2.1 \mathrm{~mm}, 5.0 \mu \mathrm{m}$ particle size, Restek) guard column. Two mobile phase components were utilized throughout the study. Mobile phase A consisted of 10 $\mathrm{mM}$ of ammonium formate in water, and mobile phase B was composed of LC-MS-grade methanol containing $0.01 \%$ tetrahydrofuran (THF). A linear gradient was programmed as $80 \%$ mobile phase $\mathrm{B}$ to $100 \% \mathrm{~B}$ over the first 5 minutes, followed by 0.5 minutes at $100 \%$ mobile phase $\mathrm{B}$, then 6 minutes at $80 \% \mathrm{~B}$, and finally 4 minutes of re-equilibration. The analysis was performed at $30^{\circ} \mathrm{C}$ with a mobile phase flow rate of $0.3 \mathrm{~mL} \cdot \mathrm{min}^{-1}$.

2) Mass spectrometry detection conditions

Mass spectral analysis was performed on an Agilent Quadrupole 1100 Mass Spectrometer fitted with electrospray ionization (ESI) source and operated in the positive ionization mode. The vaporizer was operated at $300^{\circ} \mathrm{C}$, the nebulizer gas pressure was set to $40 \mathrm{psi}$ and the capillary voltage was set to $3000 \mathrm{~V}$. The IS and UC781 were detected by their positive ion $(\mathrm{m} / \mathrm{z} 326.0$ and 336.1 , respectively) using the single ion monitoring (SIM) mode.

\subsection{Assessment of Performance Characteristics and Calculation}

\subsubsection{Linearity}

An equal weighted regression was performed to assess linearity. The deviation in the mean calculated concentrations over five runs was required to be within $15 \%$ of the nominal concentration for the non-zero calibration standards.

\subsubsection{Accuracy and Precision}

Accuracy was calculated as the percent deviation from the nominal concentrations. All intra- and inter-day precision was required to be within a coefficient of variation (CV\%) of $15 \%$ or less. Sample ranges included a low QC where the concentration was three times of the LOQ $[13,14]$, a medium QC and a high QC.

\subsubsection{Extraction Efficiency (\%)}

Extraction efficiency was calculated by dividing the area 
response of three pre-spiked QC levels (low, medium, and high in mobile phase) by the area response of extracted blank plasma that was post-spiked with the same three QC concentrations.

\subsubsection{Stability}

The stability of UC781 during sample handling was verified by subjecting samples to three freeze-thaw cycles and storage for 2 days in refrigerator $4^{\circ} \mathrm{C}$ prior to analyses. An additional test was performed to verify drug stability in the final extract for 48 hours in autosampler while tubes waiting for HPLC analysis. The samples were left at room temperature for 6 hours prior to analyses. Two concentrations medium and high (QC) samples were utilized in the stability tests.

\subsubsection{Applying the Method on the Clinical Samples}

The patient samples will be diluted 1:3 before pipetting $10 \mu \mathrm{L}$ using positive displacement pipette. An SOP was developed describing the sample collection procedure and submitted to participating clinical centers. After the measurement in order to obtain the final concentration calculated concentration will be multiplied*3.

\section{Results}

\subsection{Chromatographic Separation and Selectivity}

The approximate retention times for UC781 and IS were 2.0 and $4.3 \mathrm{~min}$, respectively. As depicted in Figures 3(a)-(c) (chromatograms of extracted drugs from CVF, with internal standard at low, medium and high QC's respectively), none of the endogenous substances from the blank CVF extracts interfered with the analyte or internal standard.

\subsection{Linearity and Limit of Quantification}

The peak area of the UC781: IS ratio for calibration standards were proportional to the level of drug in CVF over the range of tested concentrations. The calibration curves were fitted by performing weighted least-squares linear regression. The linear regression data for the calibration curves obtained from this method $(n=5)$ consistently demonstrated a coefficient of determination $\geq 0.999$. Using this method, we produced data that were linear from $50-50,000 \mathrm{ng} \cdot \mathrm{mL}^{-1}$. The low limit of quantification of UC781 was $50 \mathrm{ng} \cdot \mathrm{mL}^{-1}$. As shown in Table 1(a), this concentration demonstrated high accuracy and precision. Linearity was also tested without the internal standard to determine the direct proportionality of the UC781 peak area to the corresponding concentrations. The calculated regression coefficient $\left(\mathrm{r}^{2}\right)$ of all calibration curves was $\geq 0.999$.

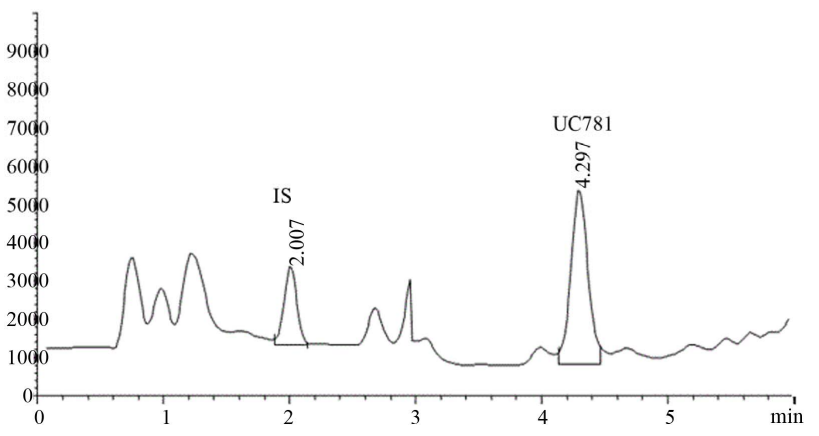

(a)

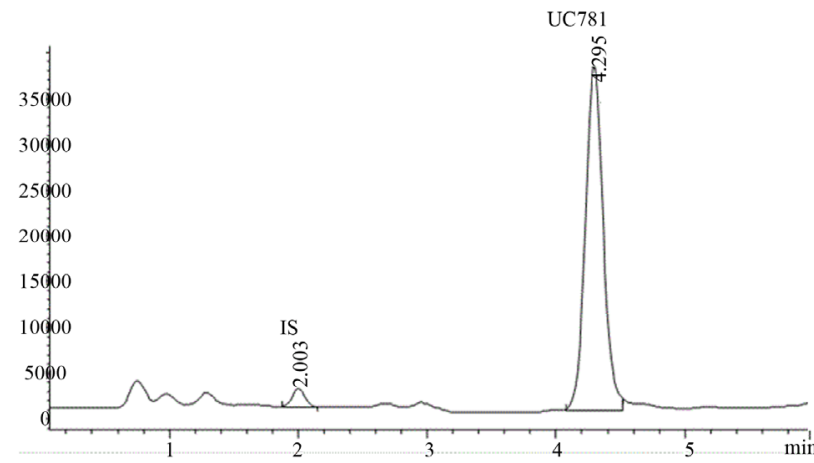

(b)

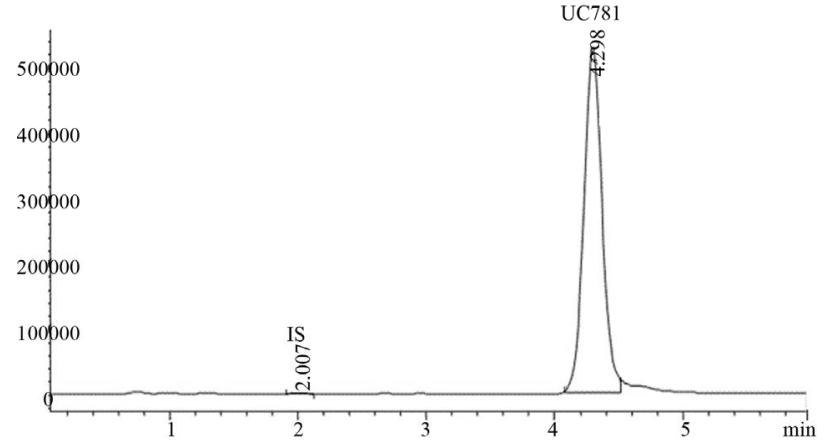

(c)

Figure 3. (a) LC-MS chromatogram of the low QC (150 ng.mL $\left.\mathrm{L}^{-1}\right)$; (b) LC-MS chromatogram of the high QC (1500 $\left.\mathrm{ng} \cdot \mathrm{mL}^{-1}\right)$; (c) LC-MS chromatogram of the high QC $(15,000$ $\left.\mathrm{ng} \cdot \mathrm{mL}^{-1}\right)$.

\subsection{Accuracy and Precision}

Results from the method validation in human CVF (Table 1) were acceptable. All observed intra- and inter-day precision (CV \%) data were at or below $15 \%$ and in accordance with the FDA guidelines [11]. Chromatograms of the three QC concentrations are illustrated in Figure 3(a) $\left(150 \mathrm{ng} \cdot \mathrm{mL}^{-1}\right)$, Figure 3(b) $\left(1500 \mathrm{ng} \cdot \mathrm{mL}^{-1}\right)$ and Figure 3(c) $\left(15,000 \mathrm{ng} \cdot \mathrm{mL}^{-1}\right)$. UC781 concentrations are presented as a percent deviation from the nominal concentrations for both within-day and between-day analyses. Using our method, precision for UC781 determinations was always $\leq 10.2 \%$ for both within- and between-day analyses. Throughout the range of control sample con- 
Table 1. Summary of accuracy and precision (\%) during method validation at low, medium and high QC concentration.

\begin{tabular}{ccccc}
\hline QC & Concentration $\left(\mathrm{ng} \cdot \mathrm{mL}^{-1}\right)$ & Accuracy $(\%)$ & Precision $(\%)$ within-day $(\mathrm{n}=5)$ & Precision $(\%)$ between-day $(\mathrm{n}=26)$ \\
\hline Low & 150 & 101.0 & 3.06 & 5.08 \\
Medium & 1500 & 102.3 & 10.20 & 6.20 \\
High & 15,000 & 99.6 & 4.60 & 6.36 \\
\hline
\end{tabular}

centrations, the intra-day precision was always lower than $10.2 \%$. Overall, the mean inter-day precision was $6.4 \%$, with mean RSDs ranging from $5.1 \%-6.4 \%$.

\subsection{Extraction Efficiency (\%)}

The extraction efficiencies for UC781 and IS from CVF using the described liquid-liquid extraction method were calculated using the ratio of the concentration of analyte in CVF to the identical concentration of the analyte prepared in mobile phase without extraction. The absolute recovery of analyte from CVF using the diethyether (liquid-liquid extraction) procedure was investigated (Table 2). This extraction method reliably eliminated interfering material from $\mathrm{CVF}$ and demonstrated high recovery $(88.3 \%)$ for three (QC) concentration levels.

\subsection{Stability}

Stability data for UC781 under various conditions are provided in Table 3. For all conditions tested, UC781 proved to be stable. All results were within the acceptance criteria of $\pm 15 \%$ deviation from the nominal concentration.

\section{Discussion}

Liquid chromatography separation hyphenated to mass spectrometry (LC-MS) has been developed into an important application in clinical pharmacology - not only for research purposes but also for routine use. At present, most important application fields are target analyses in drug measurements in a variety of biological matrices for therapeutic drug monitoring (TDM), metabolic disorders diagnosis, and many other applications. The essential strengths of LC-MS include a potentially high analytical specificity, a wide range of applicability to small or large molecules, and the opportunity to develop powerful assays with a high degree of flexibility.

There has been more than 25 million people have already died because of HIV since the discovery of the virus in 1981. This makes AIDS is one of the most disastrous epidemics in human history $[15,16]$. In year 2008 2.7 million people got infected by the HIV virus, mainly due to hetero sexual HIV transmission $[17,18]$. Despite the enormous efforts made, the development of a prophylactic AIDS-vaccine will likely not be available in the recent years to come [19]. This leads researchers to focus more on the importance of microbicides (i.e. chemical entities that can prevent or reduce the transmittance viral infection) which can applied locally (e.g. vaginally and rectally) as an alternative approach in preventing HIVtransmission [20-22].

Our research group at the University of North Carolina was first research team to focus on investigating antiretroviral (ARV); protease inhibitors (IP), nucleoside reverse transcriptase inhibitors (NRTI) and non-nucleoside reverse transcriptase inhibitors (NNRTI) in female genital tract (FGT) $[23,24]$. It was the first method to quantify drugs in direct aspirates of cervicovaginal fluids (CVF). In that study we found large deference in CVF drugs penetration (from $\leq 10 \%$ to $\geq 100 \%$ ) of blood plasma concentration [25]. Since then, we went over to the evaluation of many other ARV medications in FGT [4,26,27]. Most of these studies supported the usage of several ARV which could be an excellent pre-exposure/post-exposure prophylaxis (PrEP/PEP) candidate.

The nonnucleoside reverse transcriptase inhibitor UC781 proved to be a potential microbicide to prevent sexual transmission of human immunodeficiency virus type (HIV-1). Several gel formulations of UC781 were evaluated in a range of preclinical safety assessments, including systemic absorption analysis following topical application in human.

In this bioanalytical work, we are facing major challenges. First obstacle is the technical difficulties of UC781 in terms of solubility and stability which discussed in details elsewhere [12]. Yet, the challenge remains in dealing with CVF secretion. In brief, the vaginal fluid contains water, pyridine, squalene, urea, acetic acid, lactic acid, cholesterol, lipids, mucin, carbohydrates, amino acids, proteins, inorganic ions complex alcohols and glycols, ketones, and aldehydes. It can vary in consistency, texture, taste, color, and odor, depending on sexual arousal, the phase of the menstrual cycle, the presence of an infection, certain drugs (legal or illegal), genetic factors, and diet. Vaginal fluid is slightly acidic and can become more acidic with certain sexually transmitted diseases. The normal $\mathrm{pH}$ of vaginal fluid is between 3.8 and 4.5, whereas male semen is typically between 7.2 and 8.0 (a neutral substance has a $\mathrm{pH}$ of 7.0).

CVF is vital element of the immune system of the female genital tract. CVF is made of 1) vulvar secretions from sebaceous, sweat, Bartholins and Skene glands; 2) plasma transudate through the vaginal wall; 3) exfoliated cells; 4) bacterial products; 5) cervical mucus 6) endo- 
Table 2. Summary of the assay extraction efficiency \%.

\begin{tabular}{|c|c|c|c|c|c|c|}
\hline QC & Pre-spike & Post-spike & $\%$ recovery & Mean & SD & $\% \mathrm{CV}$ \\
\hline$\left(\mathrm{ng} \cdot \mathrm{mL}^{-1}\right)$ & Peak area & Peak area & & & & \\
\hline \multirow[t]{3}{*}{150} & 41,827 & 47,947 & 87 & 90 & 3.6 & 4.0 \\
\hline & 52,324 & 55,601 & 94 & & & \\
\hline & 52,749 & 59,237 & 89 & & & \\
\hline \multirow[t]{3}{*}{1500} & 589,493 & 648,324 & 91 & 92 & 1.7 & 1.9 \\
\hline & 516,108 & 546,940 & 94 & & & \\
\hline & 597,420 & 692,801 & 92 & & & \\
\hline \multirow[t]{3}{*}{15,000} & $5,023,683$ & $6,589,592$ & 76 & 83 & 6.2 & 7.4 \\
\hline & $5,885,670$ & $6,834,217$ & 86 & & & \\
\hline & $6,156,078$ & $7,026,467$ & 88 & & & \\
\hline
\end{tabular}

Table 3. Stability of UC781 in spiked rabbit vaginal fluid and final extract

\begin{tabular}{ccccc}
\hline Conc. $\left(\mathrm{ng} \cdot \mathrm{mL}^{-1}\right)$ & Three freeze-thaw cycles & $48 \mathrm{~h}$ at $4{ }^{\circ} \mathrm{C}$ & $8 \mathrm{~h}$ at $25^{\circ} \mathrm{C}$ & One week matrix stability \\
\hline 1500 & 10.6 & 2.5 & -2.6 & 3.3 \\
15000 & 7.7 & 1.3 & -2.8 & 5.8 \\
\hline
\end{tabular}

All values are represented as the mean of the deviation from the nominal concentration. All samples were performed in triplicate.

metrial and oviductal fluids and 7) secretions from vaginal immune cells. The latter three are influenced by sex steroid hormones e.g. during the menstrual cycle and pregnancy [28,29]. It covers the lower female genital tract $\mathrm{pH}$ and hydrates the mucosa, creating a physical barrier for microbial invasion. The important part of accurate quantification of drug in FGT is the extraction procedure.

Due to sample collection limitations with a small sample size, CVF collection is a challenging process. It was important to use dilution just right after aspiration in order to prevent fluid from forming a clot. Average sample size of normal CVF secretion ranged between 0 to 0.7 $\mathrm{mL}$. In this study, we developed and optimized liquidliquid extraction method and introduced a simple procedure allowing to accurately sampling from the collected fluid before it turns to clot. Using the positive displacement pipette with special tips (Figure 2) was important to dilute the sample before storage, similarly when transferring diluted sample for extraction. In the day of validation samples were brought to room temperature and treated for the extraction as described in the method. CVF sample dilution immediately after collection when the temperature near to $37^{\circ} \mathrm{C}$ (body temperature) is necessary, because it keeps the secretion in the fluid state. Using this procedure of sample collection and extraction, the validation data proved to be accurate and precise for any minute sample collected.

However, normal hexane was the optimal organic solvent for blood plasma [11], we found $100 \%$ of diethylether releases higher amount of UC781 compared with the other three solvents (chloroform/ether 50/50, hexane/ ether 50/50 and 100\% hexane) and SPE. This could be explained as the higher amount of lipids in blood plasma than CVF which required highly non polar solvent.

\section{Conclusion}

We have successfully developed and validated an LCMS bioanalytical method for UC781 in the CVF matrix using the sub-2 $\mu$ column with a powerful mobile phase. The method proved to be accurate meeting all validation criteria. For the rare matrix, it exhibits good linearity, precision and accuracy over a wide range of drug concentrations $\left(50-50,000 \mathrm{ng} \cdot \mathrm{mL}^{-1}\right)$. This novel sample preparation associated with liquid-liquid extraction procedure has been proved to be an excellent sample handling option for such a sensitive compound in minute amount of vaginal secretion. The method sample prep, extraction, and the powerful liquid chromatography and mass spectrometry can readily be used for accurate quantification of any similar drugs in CVF.

\section{Acknowledgements}

The authors thank the CVF donors for their participation, my analytical team at the University of North Carolina at Chapel Hill for assisting in the validation of the method, Dr. David Friend from Conrad, for providing the standard and internal standard for the method, Mrs. Soheir Hagras at the Drug Research Center, National Center of Research \& Radiation Technology (NCRRT), Egypt, and Dr. Chris Sheehan at Badirbio, Riyadh, KSA for review of this manuscript.

\section{REFERENCES}

[1] S. Taylor, M. Boffito and P. L. Vernazza, "Antiretroviral 
Therapy to Reduce the Sexual Transmission of HIV," Journal of HIV Therapy, Vol. 8, No. 3, 2003, pp. 55-66.

[2] T. Lalani and C. Hicks, "Does Antiretroviral Therapy Prevent HIV Transmission to Sexual Partners?" Current HIV/AIDS Reports, Vol. 10, No. 2, 2007, pp. 80-82. http://dx.doi.org/10.1007/s11904-007-0012-y

[3] J. M. Baeten and J. Overbaugh, "Measuirng the Infectiousness of Peresons with HIV-1: Opportunities for Preventing Sexual HIV-1 Transmission," Current HIV Research, Vol. 1, No. 1, 2003, pp. 69-86. http://dx.doi.org/10.2174/1570162033352110

[4] J. A. Talameh, N. L. Rezk and A. Kashuaba, "Quantifying the HIV-1 Integrase Inhibitor Raltegravir in Female Genital Tract Secretions Using High-Performance Liquid Chromatography with Ultraviolet Detection," Journal of Chromatography B, Vol. 878, No. 1, 2010, pp. 92-106. http://dx.doi.org/10.1016/j.jchromb.2009.11.015

[5] J. Balzarini, M. J. Perez-Perez, S. Velazquez and A. SanFelix, "Suppression of the Breakthrough of Human Immunodeficiency Virus Type 1 (HIV-1) in Cell Culture by Thiocarboxanilide Derivatives When Used Individually or in Combination with Other HIV-1-Specific Inhibitors (i.e., TSAO Derivatives)," Proceeding of the National Academy of Sciences of the United States of America, Vol. 92, 1995, pp. 5470-5474.

[6] J. Balzarini, W. G. Brouwer, D. C. Dao, E. M. Osika and E. De Clercq, "Identification of Novel Thiocarboxanilide Derivatives That Suppress a Variety of Drug-Resistant Mutant Human Immunodeficiency Virus Type 1 Strains at a Potency Similar to That for Wild-Type Virus," Antimicrobial Agents and Chemotherapy, Vol. 40, No. 6, 1996, pp. 1454-1466.

[7] R. W. J. Buckheit, T. L. Kinjerski, V. Fliakas-Boltz, J. Russell, T. L. Stup, L. A. Pallansch, et al., "StructureActivity and Cross-Resistance Evaluations of a Series of Human Immunodeficiency Virus Type-1-Specific Compounds Related to Oxathiin Carboxanilide," Antimicrobial Agents and Chemotherapy, Vol. 39, No. 12, 1995, pp. 2718-2727. http://dx.doi.org/10.1128/AAC.39.12.2718

[8] J. B. MacMahon, R. W. J. Buckheit, R. J. Gulakowski, M. J. Currens, D. T. Vistica, R. H. Schoemaker, et al., "Biological and Biochemical Anti-Human Immunodeficiency Virus Activity of UC 38, a New Non-Nucleoside Reverse Transcriptase Inhibitor," Journal of Pharmacology and Expermental Therapeutics, Vol. 276, No. 1, 1996, pp. 298-305.

[9] J. Vingerhoets, H. Azijn, E. Fransen, I. De Baere, L. Smeulders, D. Jochmans, et al., "TMC125 Displays a High Genetic Barrier to the Development of Resistance: Evidence from in Vitro Selection Experiments," Journal of Virology, Vol. 79, No. 20, 2005, pp. 12773-12782. http://dx.doi.org/10.1128/JVI.79.20.12773-12782.2005

[10] R. E. Haaland, T. Evans-Strickfaden, A. Holder, C. P. Pau, J. M. Mcnicholl, S. Chaikummaao, et al., "UC781 Microbicide Gel Retains Anti-HIV Activity in Cervicovaginal Lavag Fluids Collected Following Twice-Daialy Vaginal Application," Antimicrobial Agents and Chemotherapy, Vol. 56, No. 7, 2012, pp. 3592-3596. http://dx.doi.org/10.1128/AAC.00452-12

[11] N. Rezk, "Development and Validation of LC-ESI-MS
Method for Sensitive, Accurate and Rabid Determination of UC-781 in New Zealand White Rabbit Plasma," Talanta, Vol. 85, No. 4, 2011, pp. 2074-2079. http://dx.doi.org/10.1016/j.talanta.2011.07.037

[12] D. H. Owen and D. F. Katz, "A Vaginal Fluide Simulant," Contraception, Vol. 59, No. 2, 1999, pp. 91-95. http://dx.doi.org/10.1016/S0010-7824(99)00010-4

[13] V. P. Shah, K. K. Midha, J. W. Findlay, et al., "Bioanalytical Method Validation-Revisit with a Decade of Progress," Pharmaceutical Research, Vol. 17, No. 12, 2000, pp. 1551-1557. http://dx.doi.org/10.1023/A:1007669411738

[14] US Department of Health and Human Services, Food and Drug Administration Center for Drug Evaluation and Research (CDER), Center of Veterinary Medicine (CVM), "FDA Guidance for Industry," Pharmaceutical Research, 2001.

[15] UNAIDA, “AIDS Epidemic Update,” Geneva, 2005.

[16] UNAIDA, “AIDS Epidemic Update," Geneva, 2009.

[17] UNAIDS, "Report in the Global AIDS Epidemic," Geneva, 2008.

[18] "Will There Be an HIV Vaccine in the Next Decade?" Nature Medicine, Vol. 13, 2007, pp. 518-519. http://dx.doi.org/10.1038/nm0507-518b

[19] L. C. Rohan and A. B. Sassi, "Vaginal Drug Delivery System for HIV Prevention," American Association of Pharmaceutical Scientists, Vol. 11, No. 1, 2009, pp. 7887.

[20] B. Gulter and J. Justman, "Vaginal Microbicides and Prevention of HIV Transmission," Lancet Infection Disease, Vol. 8, No. 11, 2008, pp. 685-697.

[21] A. B. Moscicki, "Vaginal Microbicides: Where Are We and Where Are We Going?" Journal of Infection Chemotherapy, Vol. 14, No. 5, 2008, pp. 337-341. http://dx.doi.org/10.1007/s10156-008-0630-3

[22] S. M. Iqbal, T. B. Ball, P. Levinson, L. Maranan, W. Jaoko, C. Wachihi, et al., "Elevated Elafin/Trappin-2 the Female Genital Tract Is Associated with Protection against HIV Acquisition," AIDS, Vol. 23, No. 13, 2009, pp. 16691677. http://dx.doi.org/10.1097/QAD.0b013e32832ea643

[23] R. M. Grant, D. Hamer, T. Hope, R. Johnston, J. Lange, M. M. Lederman, et al., "Whither or Wither Microbicides?" Science, Vol. 321, No. 5888, 2008, pp. 532-534. http://dx.doi.org/10.1126/science.1160355

[24] S. Min, A. Corbett, N. Rezk, S. Cu-Uvinc, S. Fiscus, et al., "Protease Inhibitor and Nonnucleoside Reverse Transcriptase Inhibitor Concentration in Genital Tract of HIV1 Infected Women," Journal of Acquired Immune Deficiency Syndrome, Vol. 37, No. 5, 2004, pp. 1577-1580. http://dx.doi.org/10.1097/00126334-200412150-00008

[25] D. Julie, R. Yeh, K. Paterson, A. Corbett, B. Jung, N. Rezk, et al., "Antiretroviral Drug Exposure in the Female Genital Tract: Implications for Oral Pre- and Post Exposure Prophylaxis," AIDS, Vol. 21, No. 14, 2007, pp. 1899-1907. http://dx.doi.org/10.1097/QAD.0b013e328270385a

[26] R. Yeh, N. Rezk, A. Kashuba, et al., "Genital Tract, Cord Blood, and Amniotic Fluid Exposures of Seven Antiret- 
roviral Drugs during and after Pregnancy in Human Immunodeficiency Virus Type 1-Infected Women," Antimicrobial Agents and Chemotherapy, Vol. 53, No. 6, 2009, pp. 2367-2374. http://dx.doi.org/10.1128/AAC.01523-08

[27] A. Kawara, A. Delong, N. Rezk, et al., "Antiretroviral Drug Concentrations and HIV RNA in the Genital Tract of HIV-Infected Women Receiving Long-Term Highly Active Antiretroviral Therapy," Clinical Infectious Diseases, Vol. 46, No. 5, 2008, pp. 719-725.

http://dx.doi.org/10.1086/527387
[28] G. R. Huggins and G. Preti, "Vaginal Odors and Secretions," Clinical Obstetrics and Gynecology, Vol. 24, No. 2, 1981, pp. 355-377. http://dx.doi.org/10.1097/00003081-198106000-00005

[29] L. L. Klein, K. R. Jonschr, M. J. Heerwagen, R. S. Gibbs and J. L. McManaman, "Reprod, Shotgun Proteomic Analysis of Vaginal Fluid from Women in Late Pregnancy," Science, Vol. 15, No. 3, 2008, pp. 263-273. 